\title{
Gene-targeted-metagenomics reveals extensive diversity of aromatic dioxygenase genes in the environment
}

\author{
Shoko Iwai ${ }^{1}$, Benli Chai ${ }^{1}$, Woo Jun Sul ${ }^{1}$, James R Cole ${ }^{1}$, Syed A Hashsham ${ }^{1,2}$ and \\ James M Tiedje ${ }^{1}$ \\ ${ }^{1}$ Center for Microbial Ecology, Michigan State University, East Lansing, MI, USA and ${ }^{2}$ Department of Civil \\ and Environmental Engineering, Michigan State University, East Lansing, MI, USA
}

\begin{abstract}
Understanding the relationship between gene diversity and function for important environmental processes is a major ecological research goal. We applied gene-targeted metagenomics and pyrosequencing to aromatic dioxygenase genes to obtain greater sequence depth than possible by other methods. A polymerase chain reaction (PCR) primer set designed to target a 524-bp region that confers substrate specificity of biphenyl dioxygenases yielded 2000 and 604 sequences from the $5^{\prime}$ and $3^{\prime}$ ends of PCR products, respectively, which passed our validity criteria. Sequence alignment showed three known conserved residues, as well as another seven conserved residues not reported earlier. Of the valid sequences, $95 \%$ and $41 \%$ were assigned to 22 and 3 novel clusters in that they did not include any earlier reported sequences at $\mathbf{0 . 6}$ distance by complete linkage clustering for sequenced regions. The greater diversity revealed by this gene-targeted approach provides deeper insights into genes potentially important in environmental processes to better understand their ecology, functional differences and evolutionary origins. We also provide criteria for primer design for this approach, as well as guidance for data processing of diverse functional genes, as gene databases for most genes of environmental relevance are limited.
\end{abstract}

The ISME Journal (2010) 4, 279-285; doi:10.1038/ismej.2009.104; published online 24 September 2009

Subject Category: microbial ecology and functional diversity of natural habitats

Keywords: biphenyl; dioxygenase; gene diversity; metagenomics; pyrosequencing

\section{Introduction}

Metagenomics circumvents the problem of unculturability and has the potential to understand microbial communities at their aggregate level, transcending the individual organism to focus on the genes in a community (National Research Council, 2007). Because of the extensive genetic diversity of most microbial communities, it is currently impossible to obtain enough sequence depth to sample any gene with sufficient coverage to draw meaningful conclusions with regard to its diversity or population characteristics. This is particularly important in cases in which functional screens are problematic because of expression requirements, such as for protein complexes. To overcome this limitation, approaches are needed to target sequencing capacity to genes of particular interest. One approach is to use polymerase chain

Correspondence: JM Tiedje, Center for Microbial Ecology, Michigan State University, A540, Plant and Soil Sciences Building, East Lansing, MI 48824, USA.

E-mail: tiedjej@msu.edu

Received 15 April 2009; revised 24 August 2009; accepted 24 August 2009; published online 24 September 2009 reaction (PCR)-based targeting, together with pyrosequencing technology, similar to the manner in which it is currently carried out for 16S rRNA gene sequencing (Sogin et al., 2006; Huber et al., 2007). This approach, which we term gene-targeted metagenomics (GT-metagenomics), should then provide more extensive insight into the diversity that nature has produced, as well as provide sequence information for probes to use in recovering the entire gene from clades of interest. As many studies have shown that the mutation of a few amino acids can critically affect the structure of individual enzymes, changing their substrate utilization and degradation activities (Parales et al., 2000; Suenaga et al., 2002; Bagnéris et al., 2005; Vardar and Wood, 2005), understanding gene diversity in nature can reveal the functional, ecological and evolutionary patterns of key genes.

The GT-metagenomics approach requires that the targeted gene has sufficiently conserved regions of appropriate distance for emulsion PCR, which is required for pyrosequencing, so that primers or sets of primers will sufficiently cover a gene family. This approach is likely to be most useful for genes directly responsible for important ecosystem functions or ecological processes, such as biogeochemical 
cycles, biodegradation, pathogenesis, antibiotic resistances and cell signaling. We tested this approach on a set of dioxygenase genes important in carbon cycle for the turnover of more recalcitrant organic carbon, as well as for pollutant degradation. We also discuss requirements for primer design for GT-metagenomics and the subsequent data analysis. Our results show a much greater diversity of genes potentially important in nature's carbon cycle than realized earlier.

\section{Materials and methods}

Sample soil and DNA extraction

Polychlorinated biphenyl (PCB)-contaminated soil (15 $\mathrm{mg} \mathrm{kg}^{-1}$ ) was collected from the root zone of an Austrian pine (Pinus nigra) tree at the grounds of a paint production plant in the Czech Republic that was shown earlier to have significantly higher numbers of PCB degraders (Leigh et al., 2006). DNA was extracted as described earlier (Leigh et al., 2007) and stored at $-20^{\circ} \mathrm{C}$ until use.

\section{Primer design}

Gibson and Parales (2000) classified Rieske nonheme iron dioxygenase genes into four families: toluene/biphenyl, naphthalene, benzoate and phthalate. The Functional Gene Pipeline/Repository (FGPR) (http://fungene.cme.msu.edu/) provided the database of the sets of genes that belong to those families on the basis of monthly Hidden Markov Model (Durbin et al., 1998) searches of the DDBJ/ EMBL/GenBank non-redundant protein database (nr). Nucleotide and protein sequences of the toluene/biphenyl family of dioxygenase genes were retrieved from the FGPR bph v3.1 database (July 2007) with a cutoff score $>900$ and size $>400 \mathrm{bp}$. The 40 retrieved protein sequences with 35 different nucleotide sequences were aligned using ClustalW (Thompson et al., 1994). A PCR primer set was designed from the conserved regions of these sequences: BPHD-f3, 5'-AACTGGAARTTYGCIGC VGA-3'; BPHD-r1, 5'-ACCCAGTTYTCICCRTCGTC-3'. The specificity of the primer set was tested by comparison with the DDBJ/EMBL/GenBank database.

\section{Pyrosequencing}

PCR primers with sequencing adapter A (5'-GCCTC CCTCGCGCCATCAG- $3^{\prime}$ ) or B (5'-GCCTTGCCAGCCC GCTCAG-3') at the $5^{\prime}$ end of BPHD-f3 or BPHD-r1 were synthesized and purified by dual HPLC (Integrated DNA Technologies, Coralville, IA, USA). The PCR mixture was prepared in a total volume of $20 \mu \mathrm{l}$, containing $1 \mathrm{X}$ FastStart High Fidelity Reaction Buffer (Roche Diagnostics, Basel, Switzerland), $1.25 \mu \mathrm{M}$ of each primer, $150 \mathrm{ng} \mathrm{ll}^{-1}$ of bovine serum albumin (New England BioLabs, Ipswich, MA, USA), $0.2 \mathrm{mM}$ of dNTPs, $0.5 \mu \mathrm{l}(2.5 \mathrm{U})$ of FastStart High Fidelity PCR System Enzyme Blend (Roche Diagnostics) and 4 ng of template DNA. The
PCR condition was optimized using the genomic DNA of Burkholderia xenovorans LB400, which carries one of the target dioxygenase genes. Amplifications were performed as follows: $3 \mathrm{~min}$ at $95^{\circ} \mathrm{C}$, followed by 30 cycles of $45 \mathrm{~s}$ at $95^{\circ} \mathrm{C}, 45 \mathrm{~s}$ at $60^{\circ} \mathrm{C}$ and $40 \mathrm{~s}$ at $72^{\circ} \mathrm{C}$, then final extension for $4 \mathrm{~min}$ at $72{ }^{\circ} \mathrm{C}$. PCR products with primer pairs A-BPHD-f3 and B-BPHD-r1 or B-BPHD-f3 and A-BPHD-r1 were sequenced from $5^{\prime}$ and $3^{\prime}$ ends, respectively. Triplicate PCR products with predicted $542 \mathrm{bp}$ fragments were purified using a QIAquick Gel Extraction Kit (Qiagen, Hilden, Germany) then with a QIAquick PCR Purification Kit (Qiagen). DNA concentration was determined using a NanoDrop ND-1000 spectrophotometer (NanoDrop Technologies, Wilmington, DE, USA). Purified PCR products were mixed together with other bar-coded samples and subjected to pyrosequencing using the Genome Sequencer FLX System (454 Life Sciences, Branford, CT, USA).

\section{Determination of valid sequences}

Sequences were first trimmed of the primer region and low-quality sequences were removed as follows: We plotted the number of sequences against the first position of an ambiguous base call or stop codon. We determined the length for analysis to be at the position where the plotted curve dropped sharply (175 bp for BPHD-f3 sequences and $200 \mathrm{bp}$ for BPHD-r1 sequences). To remove possible frameshift errors, each sequence was used as a BLASTX query against a BLAST database of the translated library using a $0.001 E$-value cutoff to determine the top 10 closest matches. Those reads with the greatest number of hits that include any out-of-frame segments were successively discarded (as both query and subject), until all out-of-frame results were removed. Only the sequences that passed this filter were used for further data analysis and are termed as obtained sequences.

\section{Conservation analysis}

A total of 467 reference sequences, which have both the Rieske family domain (Pfam PF00355) and the Ring_hydroxyl_A family domain (Pfam PF00848), were retrieved from the Pfam protein family database (Finn et al., 2008). Deduced amino-acid sequences from our obtained sequences were aligned using MUSCLE (Edgar, 2004), together with the corresponding region of the reference protein sequences. Gap-treated Shannon entropy $\left(H^{\prime}\right)$ (Zhang et al., 2007) at each alignment position was calculated as:

$$
H_{i}^{\prime}=-\sum_{a=1}^{20} f_{i, a} \log _{20} f_{i, a}+f_{i, \mathrm{gap}},
$$

where $f_{i, a}$ is the relative frequency of amino acid $a$ at the alignment position $i$. $f_{i, \text { gap }}$ represents the number of gaps at the alignment position $i$ divided by the number of alignment sequences. 
Distance calculation and clustering

Dissimilarity matrices were calculated from the individual pairwise alignments of amino-acid sequences using MUSCLE with the default setting. Each value in the matrix is the fraction of the number of positions with dissimilar amino acids out of the total number of alignment positions compared, excluding end gaps. Dissimilar amino acid pairs were those for which negative probability values were assigned in BLOSUM62. These matrices were fed to DOTUR (Schloss and Handelsman, 2005) for complete linkage clustering.

Nucleotide sequence accession numbers

The nucleotide sequences described in this study have been submitted to the European Read Archive under the accession number of ERA000082.

\section{Results}

Primer design

The BPHD-f3 and BPHD-r1 primer set, corresponding to $219 \mathrm{~N}-225 \mathrm{E}$ and $387 \mathrm{D}-393 \mathrm{~V}$ of $b p h A 1$ from $B$. xenovorans LB400, respectively, was designed to amplify $524 \mathrm{bp}$ PCR products. It includes a portion of the C-terminal domain of the large oxygenase subunit that is highly diverse and has many residues responsible for congener selectivity of PCBs (Vézina et al., 2008), including four regions identified by Mondello et al. (1997). With this primer set, theoretically 31 genes out of 49 genes from bph v4.9 (FGPR, January 2009), with a cutoff score $>900$ and size $>400 \mathrm{bp}$, can be amplified and no nondioxygenase genes can be amplified using BLAST search against the DDBJ/EMBL/GenBank database. When we tried to amplify the remaining 18 genes by increasing the degeneracy of the primer set, several weak nontarget bands were observed on agarose gels, which might produce nontarget sequences by pyrosequencing (see also Discussion). These results indicated high specificity and sufficient coverage by the designed primer set. The predicted $524 \mathrm{bp}$ PCR products were successfully amplified from genomic
DNA of B. xenovorans LB400 and Rhodococcus sp. RHA1, both of which carry target dioxygenase genes, and not from genomic DNA of Sphingomonas wittichii RW1, which carries dioxin dioxygenase, which was not used for primer design.

\section{Pyrosequencing statistics}

As the average sequence length produced by the Genome Sequencer FLX System is around 200250 bp (Rothberg and Leamon, 2008), we sequenced the same pool of PCR products from both $5^{\prime}$ (BPHDf3 sequences) and $3^{\prime}$ ends (BPHD-r1 sequences) (Table 1). Although the concentration of the sequenced PCR products for BPHD-f3 and BPHD-r1 was almost the same, the number of obtained sequences differed almost threefold, possibly because of the different efficiency of emulsion PCR caused by the different primer sequences attached to the adapter. Primer-trimmed sequence length varied from 28 to $293 \mathrm{bp}$. Out of over 3000 sequences, we determined 2632 sequences $(79.3 \%)$ as obtained sequences for further analysis on the basis of our position of error and frameshift analysis. The rarefaction curves at $0,0.1,0.2,0.3,0.4,0.5$ and 0.6 clustering distances are shown in Supplementary Figure S1.

\section{Conserved regions}

We examined the conservation of residues among obtained sequences and reference sequences by calculating the Shannon entropy, $H^{\prime}$, at each alignment position. Overall patterns of conservation indicated by negative peaks of $H^{\prime}$ are similar between obtained sequences and reference sequences (Figure 1). Seven and eight highlighted residues in BPHD-f3 and BPHD-r1 alignments, respectively, were identical among $>95 \%$ of either obtained sequences or reference sequences (Figure 1). 230D, 232Y, 233H, 239H, 271G, 328P, $344 \mathrm{P}$ and $351 \mathrm{E}$, which were conserved among $>80 \%$ of reference sequences, were also highly conserved among $>98 \%$ of obtained sequences. On

Table 1 Pyrosequencing information

\begin{tabular}{|c|c|c|c|}
\hline & \multicolumn{2}{|c|}{ Sequencing primer } & \multirow[t]{2}{*}{ Sum } \\
\hline & $B P H D-f 3$ & $B P H D-r 1$ & \\
\hline Number of raw sequences & 2486 & 835 & 3321 \\
\hline Total raw sequence length & $556744 \mathrm{bp}$ & 198152 bp & 754896 bp \\
\hline Average raw sequence length & $224 \mathrm{bp}$ & $237 \mathrm{bp}$ & \\
\hline Obtained sequence ${ }^{\mathrm{a}}$ length & 175 bp (58 aа) & 200 bp (66 aа) & \\
\hline Number of obtained sequences & 2024 & 608 & 2632 \\
\hline Number of valid sequences ${ }^{\mathrm{b}}$ & 2000 & 604 & 2604 \\
\hline Unique nucleotide sequences in valid sequences & 743 & 339 & \\
\hline
\end{tabular}

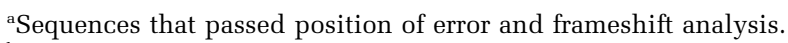

${ }^{\mathrm{b}}$ The number of sequences that have 230D, 233H and 239H for BPHD-f3 alignment and 344P for BPHD-r1 alignment. The number correspond to the position of $b p h A 1$ from $B$. xenovorans LB400. 

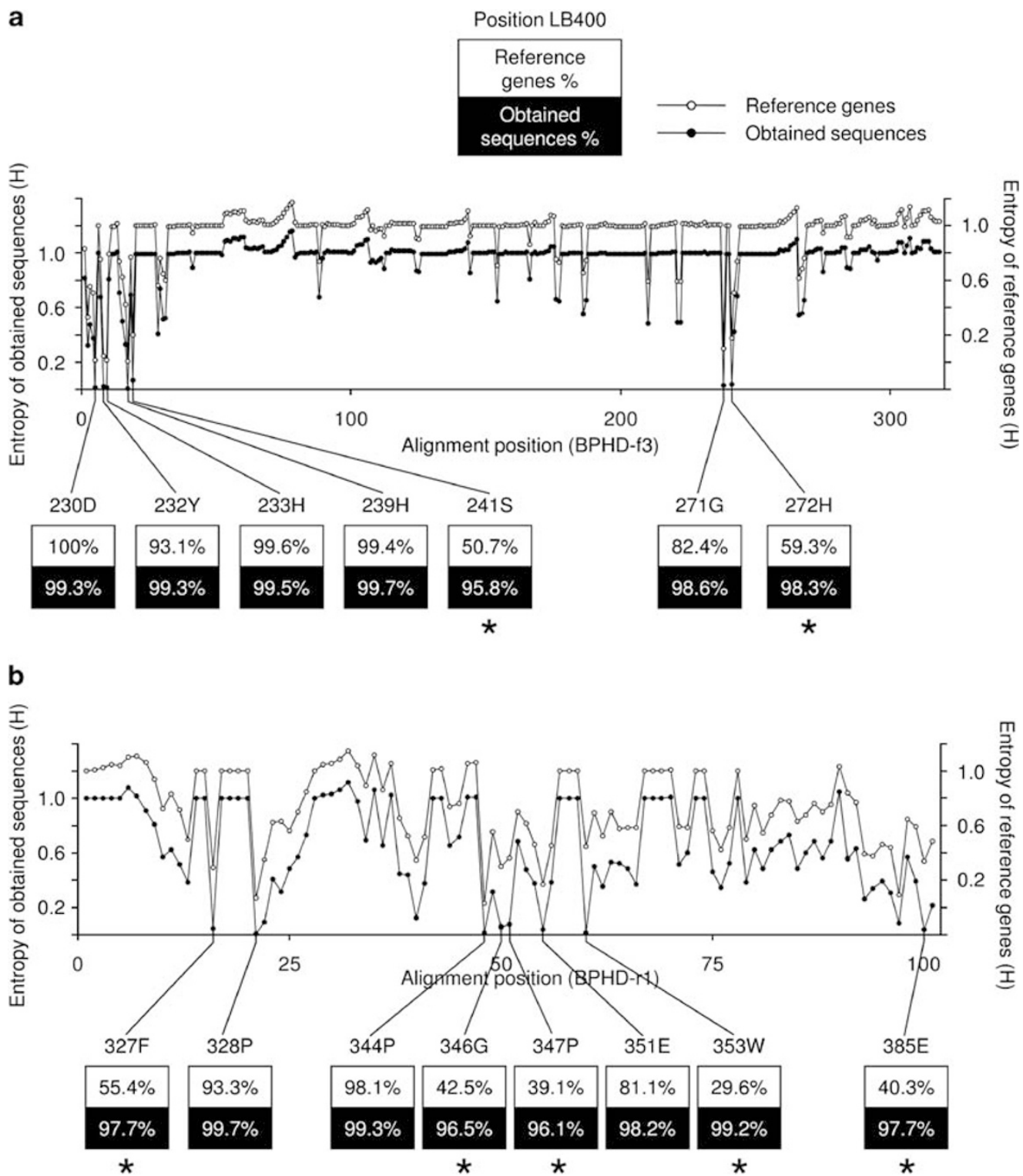

Figure 1 Shannon entropy $\left(H^{\prime}\right)$ at each alignment position and conserved residues among obtained sequences and/or reference sequences for (a) BPHD-f3 sequences and (b) BPHD-r1 sequences. Open circles $(O)$ indicate entropy of reference sequences, and filled circles ( ) indicate entropy of obtained sequences. The corresponding position numbers and the residues of bphA1 from $B$. xenovorans LB400 are indicated. The ratio of residues conserved in either set with $>95 \%$ is shown. The residues highly conserved only among obtained sequences are indicated with an asterisk.

the contrary, 241S and 272H in BPHD-f3 alignment and 327F, 346G, 347P, 353W and 385E in BPHD-r1 alignment were highly conserved among obtained sequences, but they were poorly conserved among reference sequences $(29.6-59.3 \%$ of reference sequences). On the basis of the crystal structure study, 241S, 353W and 385E are located on $\alpha$-helix 8, $\beta$-strand 19 and $\alpha$-helix 14, respectively. None of the seven residues, except $241 \mathrm{~S}$, is a part of the active site, interface residues or substrate-binding pocket (Furusawa et al., 2004).

We validated our obtained sequences using conserved residues. The highly conserved $230 \mathrm{D}, 233 \mathrm{H}$ and $239 \mathrm{H}$ (Figure 1a) are located in fragments forming the substrate-binding pocket (Furusawa et al., 2004). $233 \mathrm{H}$ and $239 \mathrm{H}$ have been reported as residues to coordinate the mononuclear iron of Rieske nonheme iron dioxygenase, and 230D acts as a bridge connecting the two iron sites, $123 \mathrm{H}$ and 233H (Furusawa et al., 2004; Dong et al., 2005). There are 24 sequences $(1.2 \%$ of the obtained sequences of BPHD-f3) that did not have one or more of those three residues, indicating that they were possible nonfunctional dioxygenase genes. Seventeen of them showed similarity with $E$-values of $<10^{-4}$ to putative dioxygenase genes from environmental samples by BLASTX. The rest of the sequences either showed similarity to nondioxygenase genes or less similarity $\left(E\right.$-value of $\left.>10^{-4}\right)$ to any of the genes in the database. As those 
sequences were missing functionally important residues, they could be caused by nonspecific PCR amplification or evolutionary degeneracies in nature. For the BPHD-r1 side, as we could not find any common structural information of the protein for this region among Rieske nonheme iron dioxygenase genes, we used highly conserved 344P (Figure 1b) for the validation of sequences. Four sequences ( $0.7 \%$ of the obtained sequences of BPHD-r1) did not have this conserved residue. All of them showed similarity with $E$-values of $<10^{-4}$ to putative dioxygenase genes from environmental samples by BLASTX. We excluded those 24 and 4 sequences that did not have the highly conserved and functionally important residues from further clustering and distribution analysis. Of the remaining 2604 sequences, which we termed as valid, 2075 sequences $(80 \%)$ have the highest BLASTX hits to dioxygenase genes (either cultured or environmental), with $E$-values of $<10^{-4}$. The remaining 529 sequences (521 sequences from BPHD-f3 and 8 sequences from BPHD-r1) have no hits with $E$-values of $<10^{-4}$ to any of the sequences in the database. The fact that those sequences have the highly conserved residues suggests that they are novel dioxygenase genes.

Clustering and distribution of the obtained sequences Those 2604 valid sequences with conserved positions were clustered together with reference sequences by complete linkage clustering on the basis of amino-acid sequences. The numbers of operational taxonomical units (OTUs) of valid sequences at each distance level are shown in Figure 2. A total of 479 and 234 unique sequences were obtained from BPHD-f3 and BPHD-r1, respectively (Figure 2).

As pyrosequencing produces a large data set, it is necessary to cluster the sequences to reduce the

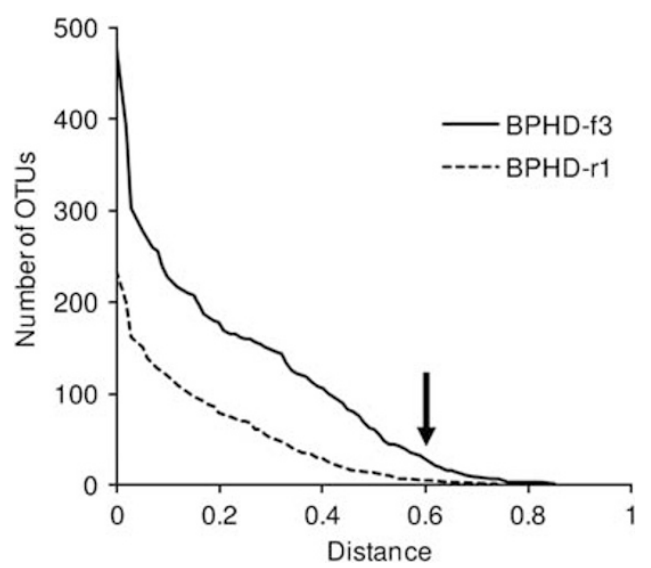

Figure 2 Clustering of valid sequences at different distance levels by complete linkage clustering on the basis of amino-acid sequences. The numbers of operational taxonomical units of valid sequences (solid line, BPHD-f3 sequences; dashed line, BPHD-r1 sequences) at each distance are shown. Arrow indicates the distance level used for distribution analysis. number for analysis. We selected 0.6 distance as a cutoff (Figure 2) to obtain 56 and 20 clusters for BPHD-f3 and BPHD-r1, respectively, numbers manageable for analysis and interpretation. Figure 3 shows the distance to the closest reference sequence(s) for each sequence in each cluster and the number of sequences in that cluster. The clusters are (i) newly obtained clusters composed of only our valid sequences (closed symbols in Figure 3) and earlier known clusters composed of (ii) both our valid sequences and reference sequences (open symbols in Figure 3), and (iii) only reference sequences. The numbers of clusters in each category are as follows: for (i), (ii) and (iii): 22, 11 and 23, respectively; and 3,4 and 13 for BPHD-f3 and BPHD-r1, respectively. Fifty-nine percent of sequences from BPHD-f3 are in the largest four clusters, which were type (i). The rest of the sequences are distributed into small clusters, which were composed of $<6 \%$ (120 sequences) of the total valid sequences. The largest type (ii) cluster from BPHD-f3 is composed of 35 sequences $(1.8 \%)$. The cluster includes toluene/biphenyl dioxygenase genes such as bphA1 from $B$. xenovorans LB400 (AAB63425) and bphAa from Rhodococcus sp. RHA1 (ABG99107) is the second largest type (ii) cluster with 18 sequences $(0.9 \%)$. Three sequences from BPHD-f3 had a similarity value of 1 to the reference sequences. For BPHD-r1 sequences, 85\% of valid sequences were in the largest two clusters and the rest were in small clusters, which have $<8 \%$ (48 sequences) of the total valid sequences. The largest cluster has 288 sequences $(48 \%)$ and was type (ii). The second largest cluster is type (i) and has 223 sequences (37\%). None of the sequences from BPHD-r1 had a similarity value of 1 to the

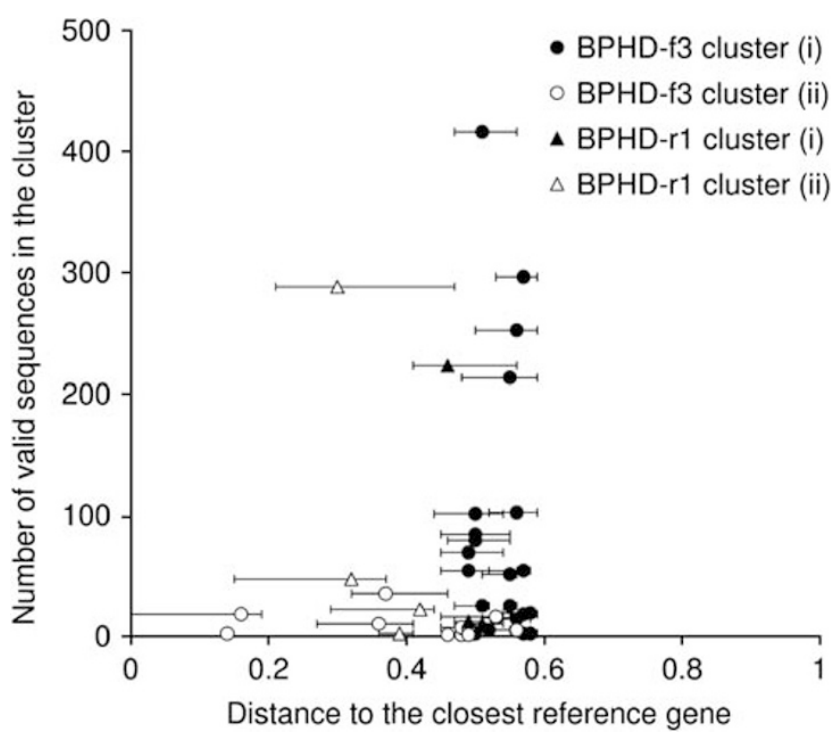

Figure 3 Pairwise distance to the closest reference sequence(s) for each valid sequence and the number of sequences in each cluster. Symbols are the median of distances in the cluster. Error bar indicates the range of the distance. 
reference sequences. The farthest distance to reference sequences was 0.59 and most sequences in novel clusters have distances between $0.45-0.6$ to reference sequences (Figure 3). Cluster distribution and frequency of each unique sequence for clusters with $>200$ sequences are shown in Supplementary Figure S2. The accession numbers of reference sequences in each cluster are shown in Supplementary Table S1.

\section{Discussion}

The particular region amplified, the degree of coverage of the target gene family and the specificity of primer sets are critical for designing PCR primer sets to assess gene populations in a community. For pyrosequencing, the length of the PCR product must not be too long or it will reduce emulsion PCR efficiency (Margulies et al., 2005). We selected the particular $524 \mathrm{bp}$ region because it provided suitably conserved primer sites, was of suitable length and covered a region known to be functionally important (confers substrate specificity). Our primer set had 48- and 16-fold degeneracy for BPHD-f3 and BPHDr1, respectively, to obtain a good coverage of target genes. As increasing degeneracy caused less specificity of the primer set, the degeneracy used here was optimal for maximizing coverage without sacrificing specificity. After we removed low-quality sequences (likely because of sequencing error and/or nondioxygenase sequences) and those without conserved positions, $80 \%$ and $72 \%$ of raw sequences remained as valid sequences for BPHD-f3 and BPHD-r1, respectively. These high ratios indicate enough primer specificity for further analysis. As a result, $95 \%$ and $41 \%$ of valid sequences were assigned to novel (type (i)) clusters. Although we designed the primers using only toluene/biphenyl dioxygenase, it is interesting that the deeper sequencing allowed us to obtain a much broader range of apparent dioxygenase genes. For example, clusters F24 and R5 contain all well-known toluene/ biphenyl dioxygenase genes and clusters F35 and R7 contain all well-known naphthalene dioxygenase genes (Supplementary Figure S2 and Supplementary Table S1). This provides a perspective on the size of clusters with and without reference sequences and hence on the diversity of the novel dioxygenase genes.

The detection of novel conserved residues, such as the seven found in this study, illustrates one of the useful outputs from the GT-metagenomics approach, in that it better reveals what nature has selected, which cannot be observed from either pure culture or shotgun metagenomics approaches. On the basis of earlier reports, six out of the seven residues have not been studied for their structural or functional importance for Rieske nonheme iron dioxygenase genes such as biphenyl dioxygenase (Furusawa et al., 2004), cumene dioxygenase (Dong et al., 2005) or naphthalene dioxygenase (Karlsson et al., 2003). However, this high conservation ratio suggests that they have some structural or functional role and hence provide new targets for site-directed mutagenesis studies. Moreover, those novel conserved regions allow us to design new primer sets to explore further diversity of genes.

The larger challenge in GT-metagenomics is data analysis. We tested several approaches. First, we selected a representative sequence from each 0.6 distance cluster on the basis of amino-acid sequences and tried to build a phylogenetic tree. However, those sequences were too diverse to produce a reliable tree (the bootstrap values were very low). We then tried a model approach to show the distribution of pyrosequenced sequences against known genes. The model approach is used to search and create databases on the basis of the relatedness to a certain group of functional genes, such as by FGPR. We built two protein models on the basis of a set of known dioxygenase genes in the toluene/ biphenyl family, and a set of all well-known Rieske nonheme iron dioxygenase genes, which includes toluene/biphenyl, naphthalene, benzoate and phthalate families. The valid sequences were searched against those models using Hidden Markov Model, and scores of each sequence to the model were calculated. Only $68 \%$ of valid sequences had scores to either of the models for BPHD-f3 sequences. In contrast, $100 \%$ of valid BPHD-r1 sequences had scores to either one of the models. This might be because the model was based on known genes and could not give similarity scores to the completely novel genes. The advantage of this method is that we can compare sequences on the basis of the score to the model, regardless of region and length. When there is enough reference gene information, for example, by adding new clusters obtained in this and other studies, to provide a more stable model, the model approach will be a useful method to map and compare sequences obtained from different regions of the gene.

The most abundant cluster in BPHD-r1 sequences was the novel (type (i)) cluster with $48 \%$ of total valid sequences (Figure 3). However, there is no such large type (i) cluster in BPHD-f3. In the same manner, we found differences between BPHD-f3 and BPHD-r1 clustering in terms of the proportion of clusters in each type. This suggests a further complexity of environmental dioxygenases composed of different combinations of those clusters in one gene. Advances in sequencing technology that provide greater read length, such as the recently introduced GS FLX Titanium Series, which can extend read length to 400-500 bp (http://www.454. $\mathrm{com} /$ ), will be a great help with GT-metagenomics approaches.

A this GT-metagenomics approach is based on PCR products, it, similar to all PCR-based approaches, should be assumed to have primer bias and cannot be comprehensive or reflect actual 
quantification. It can, however, reveal much more sequence information about the genes it does recover and hence more insight into what nature has produced. Functional genes often have less conserved regions and the current sequenced length is less than desired. Thus, using several different primer sets, which can be designed from new conserved regions, and combining information from the model approach, should help to better understand the biology behind gene diversity. This information should also provide the probes or other tools that can aid the recovery of full-length gene sequences from the environment for functional studies. Moreover, testing the expression and activity of those genes toward aromatic hydrocarbons will be important for suggesting their function in nature.

\section{Acknowledgements}

We thank Chike V Anadumaka for technical assistance, and acknowledge the important work by Mary Beth Leigh, Martina Mackova, Tomas Macek and Ondrej Uhlik, which established this rhizosphere site as important for aromatic biodegradation studies. This study was supported by the NIEHS grant under the Superfund Basic Research Program 5P42 ES004911-18/19.

\section{References}

Bagnéris C, Cammack R, Mason JR. (2005). Subtle difference between benzene and toluene dioxygenases of Pseudomonas putida. Appl Environ Microbiol 71: 1570-1580.

Dong XS, Fushinobu S, Fukuda E, Terada T, Nakamura S, Shimizu K et al. (2005). Crystal structure of the terminal oxygenase component of cumene dioxygenase from Pseudomonas fluorescens IP01. J Bacteriol 187: $2483-2490$.

Durbin R, Eddy S, Krogh A, Mitchison G. (1998). Biological Sequence Analysis: Probabilistic Models of Proteins and Nucleic Acids. Cambridge University Press: UK.

Edgar RC. (2004). MUSCLE: multiple sequence alignment with high accuracy and high throughput. Nucleic Acids Res 32: 1792-1797.

Finn RD, Tate J, Mistry J, Coggill PC, Sammut SJ, Hotz HR et al. (2008). The Pfam protein families database. Nucleic Acids Res 36: D281-D288.

Furusawa Y, Nagarajan V, Tanokura M, Masai E, Fukuda M, Senda T. (2004). Crystal structure of the terminal oxygenase component of biphenyl dioxygenase derived from Rhodococcus sp. strain RHA1. J Mol Biol 342: 1041-1052.

Gibson DT, Parales RE. (2000). Aromatic hydrocarbon dioxygenases in environmental biotechnology. Curr Opin Biotechnol 11: 236-243.

Huber JA, Mark Welch D, Morrison HG, Huse SM, Neal PR, Butterfield DA et al. (2007). Microbial population structures in the deep marine biosphere. Science 318: 97-100.
Karlsson A, Parales JV, Parales RE, Gibson DT, Eklund H, Ramaswamy S. (2003). Crystal structure of naphthalene dioxygenase: side-on binding of dioxygen to iron. Science 299: 1039-1042.

Leigh MB, Pellizari VH, Uhlík O, Sutka R, Rodrigues J, Ostrom NE et al. (2007). Biphenyl-utilizing bacteria and their functional genes in a pine root zone contaminated with polychlorinated biphenyls (PCBs). ISME J 1: 134-148.

Leigh MB, Prouzová P, Macková M, Macek T, Nagle DP, Fletcher JS. (2006). Polychlorinated biphenyl (PCB)-degrading bacteria associated with trees in a PCB-contaminated site. Appl Environ Microbiol 72: 2331-2342.

Margulies M, Egholm M, Altman WE, Attiya S, Bader JS, Bemben LA et al. (2005). Genome sequencing in microfabricated high-density picolitre reactors. Nature 437: $376-380$.

Mondello FJ, Turcich MP, Lobos JH, Erickson BD. (1997). Identification and modification of biphenyl dioxygenase sequences that determine the specificity of polychlorinated biphenyl degradation. Appl Environ Microbiol 63: 3096-3103.

National Research Council (2007). Why Metagenomics? In: The New Science of Metagenomics. The National Academies Press: Washington DC, pp 12-32.

Parales RE, Lee K, Resnick SM, Jiang H, Lessner DJ, Gibson DT. (2000). Substrate specificity of naphthalene dioxygenase: effect of specific amino acids at the active site of the enzyme. J Bacteriol 182: 1641-1649.

Rothberg JM, Leamon JH. (2008). The development and impact of 454 sequencing. Nat Biotechnol 26: 1117-1124.

Schloss PD, Handelsman J. (2005). Introducing DOTUR, a computer program for defining operational taxonomic units and estimating species richness. Appl Environ Microbiol 71: 1501-1506.

Sogin ML, Morrison HG, Huber JA, Welch DM, Huse SM, Neal PR et al. (2006). Microbial diversity in the deep sea and the underexplored 'rare biosphere'. Proc Natl Acad Sci USA 103: 12115-12120.

Suenaga H, Watanabe T, Sato M, Ngadiman, Furukawa K. (2002). Alteration of regiospecificity in biphenyl dioxygenase by active-site engineering. J Bacteriol 184: $3682-3688$.

Thompson JD, Higgins DG, Gibson TJ. (1994). CLUSTAL W: improving the sensitivity of progressive multiple sequence alignment through sequence weighting, position-specific gap penalties and weight matrix choice. Nucleic Acids Res 22: 4673-4680.

Vardar G, Wood TK. (2005). Protein engineering of toluene-o-xylene monooxygenase from Pseudomonas stutzeri OX1 for enhanced chlorinated ethene degradation and o-xylene oxidation. Appl Microbiol Biotechnol 68: 510-517.

Vézina J, Barriault D, Sylvestre M. (2008). Diversity of the C-terminal portion of the biphenyl dioxygenase large subunit. J Mol Microbiol Biotechnol 15: 139-151.

Zhang SW, Zhang YL, Pan Q, Cheng YM, Chou KC. (2007). Estimating residue evolutionary conservation by introducing von Neumann entropy and a novel gap-treating approach. Amino Acids 35: 495-501.

Supplementary Information accompanies the paper on The ISME Journal website (http://www.nature.com/ismej) 